\title{
THE INFLUENCE OF "INJECTED" AND "THERMAL" MAGNONS ON A SPIN WAVE CURRENT AND DRAG EFFECT IN HYBRID STRUCTURES
}

\author{
Igor Lyapilin ${ }^{1,2}$ and Mikhail Okorokov ${ }^{1}$ e-mail: Okorokovmike@gmail.com \\ ${ }^{1}$ Institute of Metal Physics, Ural Branch of the Russian Academy of Sciences, ul. S. Kovalevskoi 18, Yekaterinburg, 620990 \\ Russia \\ ${ }^{2}$ Ural Federal University, ul. Mira 19, Yekaterinburg, 620002 Russia
}

\begin{abstract}
The formation of the two: injected and thermally excited, different in energies magnon subsystems and the influence of its interaction with phonons and between on drag effect under spin Seebeck effect conditions in the magnetic insulator part of the metal/ferromagnetic insulator/metal structure is studied. The analysis of the macroscopic momentum balance equations of the systems of interest conducted for different ratios of the drift velocities of the magnon and phonon currents show that the injected magnons relaxation on the thermal ones is possible to be dominant over its relaxation on phonons. This interaction will be the defining in the forming of the temperature dependence of the spin-wave current under spin Seebeck effect conditions, and inelastic part of the magnon-magnon interaction is the dominant spin relaxation mechanism.
\end{abstract}

\section{Introduction}

Recently, a great deal of attention is devoted to the investigation of thermally excited magnons, particularly in studies of the spin Seebeck effect (SSE) in $\mathrm{Pt} / \mathrm{YIG} / \mathrm{Pt}$ structures. The propagation of magnons in a magnetic insulator is described by two characteristic quantities: mean free path and spin diffusion length that are governed, in turn, by various magnon relaxation mechanisms. A series of experiments determine the range of the diffusion lengths as being quite wide: from $4 \mu \mathrm{m}$ to $120 \mu \mathrm{m}$ [1-5]. To explain so large values of the spin diffusion lengths, the number of papers has put forward several concepts of appearance, along with thermal magnons, of long-wave subthermal magnons in a magnetic insulator. For interpreting the experimental results, the works $[4,5]$ have adopted the hypothesis of the existence of the two magnon subsystems with different energies: thermal and subthermal magnons in a magnetic insulator. As to the temperature dependence of the Seebeck coefficient, it is non-monotonic and reaches its maximum within the range of $50-100 \mathrm{~K}$. And as the investigations have shown, it is affected by strength of a magnetic field, dimensions of the samples, and quality of the interface [5]. To explain the low temperature enhancement proposed the phonon-drag SSE scenario based on a theoretical model [6-8]. However, the observed transport of magnons over a long distance of up to millimeters in magnetic insulators implies a relative weak interaction with phonons and impurities, and the measurements of the temperature dependent thermal conductance of YIG single crystals show that the phonon contribution to the thermal conductivity reaches its maximum at around $25 \mathrm{~K}$, which is $50 \mathrm{~K}$ lower than the observed peak in the SSE.

We have developed an "enhanced model" of the spin current and drag effect, which is based on the formation of two interacting magnon flows. Indeed, when SSE occurs in such structures, there are two magnon groups with different energies. The first group consists of magnons produced by an inhomogeneous temperature field applied to the magnetic insulator- "thermal" magnons. The energy of the magnons is of the order of a temperature $\left(k_{B} T\right)$. Along with them, there are the magnons injected into the magnetic insulator due to inelastic scattering of spin-polarized electrons of the metal by localized spins located in the vicinity of the interface. The energy of the "injected" magnons is of the order of spin accumulation energy of conduction electrons of the metal $\Delta_{s}>k_{B} T$. Thus, it can be said that the magnetic system of the insulator forms another subsystem of "injected" magnons that are actually responsible for the SSE. As a consequence, in the presence of a non-uniform temperature field, there are three flows inside the magnetic insulator, namely, phonon and two magnon ones. The evolution of the magnon and phonon subsystems to equilibrium occurs due to the relaxation of both their energy and their moment. Obviously, the interaction between the flows gives rise to the drag effect. 


\section{Macroscopic momentum balance equations}

Under the influence of a non-uniform temperature field (a temperature gradient) applied to the system, the magnons and phonons begin travelling; their macroscopic drift affects the propagation of the spinwave current. The problem to be solved reduces to constructing and analyzing a set of macroscopic momentum balance equations for the magnon $(i=1,2)$ and phonon $(i=p)$ subsystems. To derive the macroscopic equations

$$
\left\langle\dot{P}_{i}(\mathbf{r})\right\rangle^{t}=S p\left\{\dot{P}_{i}(\mathbf{r}) \rho(t)\right\},\left(i=m_{1}, m_{2}, p\right)
$$

The expression for the non-equilibrium statistical operator (NSO) needs to be sought. According to [9], for $\rho(t)$, in the linear approximation in deviation from equilibrium, we have:

$\rho(t)=\rho_{q}(t)-\int_{-\infty}^{0} d t^{\prime} e^{\epsilon t^{\prime}} e^{i t^{\prime} L} \int_{0}^{1} d \tau \rho_{0}^{\tau} \dot{S}\left(t+t^{\prime}\right) \rho_{0}^{-\tau} \rho_{0}$.

$\dot{S}(t)=\partial S(t) / \partial t+(i \hbar)^{-1}[S(t), H]$ is the entropy production operator. $e^{i t L} A=e^{-i t H / \hbar} A e^{i t H / \hbar}, \rho_{0}=$ $\exp \left\{-S_{0}\right\} . S_{0}$ is the entropy operator for the equilibrium system. Thus, the problem boils down to finding the entropy production operator. We can write the entropy operator as

$$
\begin{aligned}
& S(t)= \Phi(t)+\int d \mathbf{r}\left\{\beta _ { m _ { i } } ( \mathbf { r } , t ) \left[H_{m_{i}}(\mathbf{r})+H_{m_{i} m_{i}}(\mathbf{r})+\right.\right. \\
&\left.+H_{m_{i} m_{j}}(\mathbf{r})\right]-\beta \mu_{m_{i}}(\mathbf{r}, t) N_{m_{i}}(\mathbf{r})+ \\
&+ \beta_{p}(\mathbf{r}, t)\left[H_{p}(\mathbf{r})+H_{p p}(\mathbf{r})+H_{p m_{i}}(\mathbf{r})\right]- \\
&\left.-\beta_{m_{i}}(\mathbf{r}, t) V_{m_{i}}(\mathbf{r}, t) P_{m_{i}}(\mathbf{r})+\beta_{p}(\mathbf{r}, t) V_{p}(\mathbf{r}, t) P_{p}(\mathbf{r})\right\}
\end{aligned}
$$

Here

$$
\begin{gathered}
I_{m_{i}}^{*}(\mathbf{r})=\left[I_{H_{m_{i}}}(\mathbf{r})+I_{P_{m_{i}}}(\mathbf{r}) V_{m_{i}}(\mathbf{r})\right], \\
I_{p}^{*}(\mathbf{r})=\left[I_{H_{p}}(\mathbf{r})+V_{p}(\mathbf{r}) I_{P_{p}}(\mathbf{r})\right]
\end{gathered}
$$

the density flows of the energy and momentum magnons and phonons. We also have taken into account that $\nabla\left(\beta_{k}(\mathbf{r}, t) V_{k}(\mathbf{r}, t)\right) \sim V_{k}(t) \nabla \beta_{k}(\mathbf{r}, t)$. Here $\beta_{m_{i}}(\mathbf{r}, t)$ are local-equilibrium values of the inverse temperatures of the magnon $(i=1,2)$ and phonon subsystems $\beta_{p}(\mathbf{r}, t) . \quad \mu_{m_{i}}(\mathbf{r}, t)$ is a local equilibrium value of the chemical potential of the magnons. $N(\mathbf{r})=N_{m_{1}}(\mathbf{r})+N_{m_{2}}(\mathbf{r})$ is the magnon number density operator. $V_{m_{i}}, V_{p}$ are the drift velocities of the magnons $(i=1,2)$ and the phonons respectively.

Inserting the entropy production operator (3) into the expression for the NSO (2), we average the operator equations (1) for momenta of the subsystems under discussion. Then we have

$$
\begin{aligned}
& \left\langle\dot{P}_{m_{1}}(\mathbf{r})\right\rangle^{t}=-\int_{-\infty}^{0} d t^{\prime} e^{\epsilon t^{\prime}} \int d \mathbf{r}^{\prime} \beta\left\{D_{m_{1} m_{1}}\left(\mathbf{r}, \mathbf{r}^{\prime}, t^{\prime}\right) \nabla \mu_{m_{1}}\left(\mathbf{r}^{\prime}, \bar{t}\right)+\right. \\
& +L_{\left(m_{1}, m_{1} p\right)}\left(\mathbf{r}, \mathbf{r}^{\prime}, t^{\prime}\right) \delta V_{m_{1}, p}\left(\mathbf{r}^{\prime}, \bar{t}\right)+ \\
& \left.+L_{\left(m_{1}, m_{1} m_{2}\right)}\left(\mathbf{r}, \mathbf{r}^{\prime}, t^{\prime}\right) \delta V_{m_{1}, m_{2}}\left(\mathbf{r}^{\prime}, \bar{t}\right)\right\}, \\
& \left\langle\dot{P}_{m_{2}}(\mathbf{r})\right\rangle^{t}=-\int_{-\infty}^{0} d t^{\prime} e^{\epsilon t^{\prime}} \int d \mathbf{r}^{\prime} \beta\left\{D_{m_{2} m_{2}}\left(\mathbf{r}, \mathbf{r}^{\prime}, t^{\prime}\right) \nabla \mu_{m_{2}}\left(\mathbf{r}^{\prime}, \bar{t}\right)+\right. \\
& +L_{\left(m_{2}, m_{2} p\right)}\left(\mathbf{r}, \mathbf{r}^{\prime}, t^{\prime}\right) \delta V_{m_{2}, p}\left(\mathbf{r}^{\prime}, \bar{t}\right)+ \\
& \left.+L_{\left(m_{2}, m_{1} m_{2}\right)}\left(\mathbf{r}, \mathbf{r}^{\prime}, t^{\prime}\right) \delta V_{m_{2}, m_{1}}\left(\mathbf{r}^{\prime}, \bar{t}\right)\right\},
\end{aligned}
$$

here $\bar{t} \equiv t+t^{\prime}$. The first summands in the right-hand side of (4), (5) describe magnon-diffusion processes due to inhomogenius magnon chemical potential. The last two - magnon-phonon and "thermal" - "coherent" magnons scattering respectively

$$
\begin{aligned}
\left\langle\dot{P}_{p}(\mathbf{r})\right\rangle^{t} & =-\int_{-\infty}^{0} d t^{\prime} e^{\epsilon t^{\prime}} \int d \mathbf{r}^{\prime} \beta\left\{-D_{p p}\left(\mathbf{r}, \mathbf{r}^{\prime}, t^{\prime}\right) \nabla \beta_{p}\left(\mathbf{r}^{\prime}, \bar{t}\right)+\right. \\
+ & L_{\left(p, m_{1} p\right)}\left(\mathbf{r}, \mathbf{r}^{\prime}, t^{\prime}\right) \delta V_{p, m_{1}}\left(\mathbf{r}^{\prime}, \bar{t}\right)+ \\
+ & L_{\left(p, m_{2} p\right)}\left(\mathbf{r}, \mathbf{r}^{\prime}, t^{\prime}\right) \delta V_{p, m_{2}}\left(\mathbf{r}^{\prime}, \bar{t}\right)+ \\
& \left.+L_{(p, p p)}\left(\mathbf{r}, \mathbf{r}^{\prime}, t^{\prime}\right) V_{p}\left(\mathbf{r}^{\prime}, \bar{t}\right)\right\} .
\end{aligned}
$$

The expression (6 ) describes the diffusive processes, processes of non-magnon relaxation of phonons and the magnon-phonon scattering. Here we introduce the notations: $\delta V_{i k}=V_{i}-V_{k}, \quad D_{p p}\left(\mathbf{r}, \mathbf{r}^{\prime}, t^{\prime}\right)=$ $\beta\left(\nabla I_{P_{p}}(\mathbf{r}), I_{p}^{*}\left(\mathbf{r}^{\prime}, t^{\prime}\right)\right)$ and

$$
(A, B)=\int_{0}^{1} d \lambda S p\left\{A \rho_{0}^{\lambda} \Delta B \rho_{0}^{1-\lambda}\right\} .
$$

The momentum balance equations allow conducting the analysis of how the interaction between the subsystems at hand affects the implementation of the drag effect. We introduce the average values of the forces induced by the chemical potential and temperature gradients

$$
\begin{gathered}
F_{m_{i}}(\mathbf{r})=\int_{-\infty}^{0} d t^{\prime} e^{\epsilon t^{\prime}} \int d \mathbf{r}^{\prime} D_{m_{i} m_{j}}\left(\mathbf{r}, \mathbf{r}^{\prime}, t^{\prime}\right) \nabla \mu_{m_{j}}\left(\mathbf{r}^{\prime}, \bar{t}\right), \\
F_{p}(\mathbf{r})=\int_{-\infty}^{0} d t^{\prime} e^{\epsilon t^{\prime}} \int d \mathbf{r}^{\prime} D_{p p}\left(\mathbf{r}, \mathbf{r}^{\prime}, t^{\prime}\right) \nabla \beta_{p}\left(\mathbf{r}^{\prime}, \bar{t}\right) .
\end{gathered}
$$

Besides, introduce the inverse times of the magnon and phonon momentum relaxation caused by interaction with phonons processes of non-magnon relaxation of phonons. Let us designate them as $\omega_{(m p)}$, and $\omega_{(p p)}$, respectively [9]

$$
\omega_{(\gamma, v)}=\left(P_{\gamma}, P_{\gamma}\right)^{-1} \int_{-\infty}^{0} d t^{\prime} e^{\epsilon t^{\prime}}\left(\dot{P}_{(\gamma, v)}, \dot{P}_{(\gamma, v)}\left(t^{\prime}\right)\right),
$$


$\gamma=m_{1}, m_{2}, p$. We restrict ourselves to the discussion of a stationary case. For this purpose, we average the balance equations over time $t$. To start the analysis, we consider the simplest case when the drift velocities of the magnon systems are equal: $V_{m_{1}}=V_{m_{2}} \equiv V_{m}$ and $\beta_{m_{1}}=\beta_{m_{2}}$. The balance equation for the magnon momentum acquires the form

$$
F_{m}=V_{m} \mathcal{P}_{m} \frac{\omega_{(p, p p)} \omega_{(m, m p)}}{\omega_{(m, m p)}+\omega_{(p, p p)}} \equiv V_{m} \mathcal{P}_{m} \Omega .
$$

where $\mathcal{P}_{m} \equiv\left(P_{m}, P_{m}\right), \quad \mathcal{P}_{p} \equiv\left(P_{p}, P_{p}\right)$. Finally, the dragging leads to the change in frequency of the magnon-phonon collisions, and the quantity $\Omega$ is the inverse relaxation time of the magnon momentum by non-equilibrium phonons. From the expression (7) it follows that the drag effect has an influence on the magnon-phonon collision frequency. The criterion of realizing the drag effect consists in the requirement $\omega_{(m, m p)}>\omega_{(p, p p)}$ that coincides with the solution of the kinetic equation [10].

Another limiting case corresponds to the situation when the drift velocities of thermal magnons and phonons are equal to $V_{m_{2}}=V_{p}$ and $\left(\beta_{m_{2}}=\beta_{p}\right)$. In this case, thermal magnons and phonons form one subsystem. From balance equations we obtain

$$
F_{m_{1}}=V_{m_{1}} \frac{\omega_{(p, p p)}\left[\omega_{(p, m p)}+\omega_{\left(m, m_{1} m_{2}\right)}\right]}{\omega_{(p, m p)}+\omega_{(p, p p)}} .
$$

From the expression (8) it follows that if $\omega_{(p, p p)} \gg$ $\omega_{(p, m p)}$ then $F_{1} \sim \omega_{(p, m p)}$ and $F_{1} \sim \omega_{\left(m, m_{1} m_{2}\right)}$ if $\omega_{(p, m p)} \ll \omega_{\left(m, m_{1} m_{2}\right)}$. If the opposite inequality, when $\omega_{(p, p p)} \ll \omega_{(p, m p)}$ then $F_{1} \sim \omega_{(p, p p)}[1+$ $\left.\omega_{\left(m, m_{1} m_{2}\right)} / \omega_{(p, m p)}\right]$ and $F_{1} \sim \omega_{(p, p p)}$ if $\omega_{\left(m, m_{1} m_{2}\right)} \ll$ $\omega_{(p, m p)}$ and $F_{1} \sim \omega_{(p, p p)} \omega_{\left(m, m_{1} m_{2}\right)} / \omega_{(p, m p)}$ when $\omega_{\left(m, m_{1} m_{2}\right)} \gg \omega_{(p, m p)}$.

Now we look into the drag effect in the event of two magnon and one phonon systems. Then, the momentum balance equations can be written as follows. The set of the equations (5), (6) implies

$$
\begin{gathered}
F_{m_{1}}=\left\{\omega_{\left(m_{1}, m p\right)}+\omega_{\left(m, m_{1} m_{2}\right)}-\frac{\omega_{\left(m_{1}, m p\right)} \omega_{\left(m_{1}, m p\right)}}{\Omega}\right\} V_{m_{1}} \\
-\left\{\frac{\omega_{\left(m_{1}, m p\right)} \omega_{\left(m_{2}, m p\right)}}{\Omega}+\omega_{\left(m, m_{1} m_{2}\right)}\right\} \\
\cdot\left\{\frac{F_{m_{2}}+\left(\omega_{\left(m, m_{1} m_{2}\right)}+\omega_{\left(m_{1}, m p\right)} \omega_{\left(m_{2}, m p\right)} / \Omega\right) V_{m_{1}}}{\omega_{\left(m_{2}, m p\right)}+\omega_{\left(m, m_{1} m_{2}\right)}-\omega_{\left(m_{2}, m p\right)} \omega_{\left(m_{2}, m p\right)} / \Omega},\right.
\end{gathered}
$$

where $\Omega=\omega_{\left(m_{1}, m p\right)}+\omega_{\left(m_{2}, m p\right)}+\omega_{(p, p p)}$.

Let the energy transfer channels from the magnon subsystems to the phonon subsystem be equal $\omega_{\left(m_{1}, m p\right)}=\omega_{\left(m_{2}, m p\right)}=\omega_{(m, m p)}, V_{m_{1}}=V_{m}$. In this case we have

$$
\begin{gathered}
F_{m_{1}}=\left\{\omega_{(m, m p)}+\omega_{\left(m, m_{1} m_{2}\right)}-\omega_{(m, m p)} / \Omega\right\} V_{m} \\
-\left\{\omega_{(m, m p)} / \Omega+\omega_{\left(m, m_{1} m_{2}\right)}\right\} \times \\
\times\left\{\frac{F_{m_{2}}+\left(\omega_{\left(m, m_{1} m_{2}\right)}+\omega_{(m, m p)} / \Omega\right) V_{m}}{\omega_{(m, m p)}+\omega_{\left(m, m_{1} m_{2}\right)}-\omega_{(m, m p)} / \Omega}\right\} .
\end{gathered}
$$

Here $\Omega=2+\omega_{(p, p p)} / \omega_{(m, m p)}$. If $\omega_{(p, p p)} \gg \omega_{(m, m p)}$, then

$$
\begin{aligned}
& F_{m_{1}}=\left\{\omega_{(m, m p)}+\omega_{\left(m, m_{1} m_{2}\right)}\right\} V_{m}- \\
&-\omega_{\left(m, m_{1} m_{2}\right)} \cdot\left\{\frac{F_{m_{2}}+\omega_{\left(m, m_{1} m_{2}\right)}}{\omega_{(m, m p)}+\omega_{\left(m, m_{1} m_{2}\right)}} .\right.
\end{aligned}
$$

The expression (11) claims that the spin-wave current $\sim F_{1}$ is determined by the relations between the correlation functions $\omega_{\left(m, m_{1} m_{2}\right)}$ and $\omega_{(m, m p)}$. As it follows from the expression (11) that if $\omega_{\left(m, m_{1} m_{2}\right)} \ll \omega_{(m, m p)}$ then $F_{1} \sim \omega_{(m, m p)}$. In this case magnon-phonon interaction is the dominant channel of a magnon relaxation. If we have the opposite inequality $\omega_{\left(m, m_{1} m_{2}\right)} \gg$ $\omega_{(m, m p)}$ then $F_{1} \sim \omega_{\left(m, m_{1} m_{2}\right)}$. In this case the interaction between "injected" and "thermal" magnons is the dominant channel of a magnon relaxation. Moreover, the inelastic scattering of the "injected" magnons by "thermal" ones can be regarded as scattering by impurity centers whose concentration is temperature-varied. This interaction will determine the temperature-field behavior of the spin-wave current under the conditions of the Seebeck spin effect.

Conclusion. The analysis of the macroscopic momentum balance equations of the systems of interest conducted for different ratios of the drift velocities of the magnon and phonon currents show that the "injected" magnons relaxation on the "thermal" ones is possible to be dominant over its relaxation on the phonons. This interaction will be the defining in the forming of the temperature dependence of the spinwave current under SSE conditions, and inelastic part of the magnon-magnon interaction is the dominant spin relaxation mechanism.

Acknowledgments. The given work has been done as the part of the state task on the theme "Electron" 01201463330 (project 12-T-2-1011) with the support of the Ministry of Education of the Russian Federation (Grant 14.Z50.31.0025, 16-02-00044) and project no. $15-17-2-17)$.

\section{References}

[1] S. R. Boona, J. P. Heremans, Phys. Rev. B 90, 064421 (2014).

[2] L. J. Cornelissen, J. Liu, R. A. Duine, J. B. Youssef, B. J. van Wees, Natur. Phys. 11, 1022 (2015).

[3] B. L. Giles, Z. Yang, J. S. Jamison, R. C. Myers, Phys. Rev. B 92, 224415 (2015).

[4] A. Kehlberger, U. Ritzmann, D. Hinzke, E. J. Guo, J. Cramer, G. Jakob, M. C. Onbasli, D. H. Kim, C. A. Ross, M. B. Jungfleisch, B. Hillebrands, U. Nowak, M. Kleaui, Phys, Rev. Lett. 115, 096602 (2015).

[5] T. Kikkawa, K. Uchida, S. Daimon, Y. Shiomi, H. Adachi, Z. Qiu, D. Hou, X. F. Jin, S. Maekawa, E. Saitoh, Phys. Rev. B 88, 214403 (2013).

[6] L. E. Gurevich and G. A. Roman, Sov. Phys. Solid State 8, 2102 (1967). 
[7] F. J. Blatt, P. A. Schroeder, C.L. Foiles and D. Greig, Thermoelectric Power of Metals (New York: Plenum 1976).

[8] M. Bailyn, Phys. Rev. 126, 2040 (1962).
[9] I.I. Lyapilin, M.S. Okorokov, V. V. Ustinov, Phys.Rev. B 91, 195309 (2015).

[10] R. J. Kubo, Peport on Progr in Phys. 29, 1 (1966). 